\title{
Impact of Digital Social Media on Indian Higher Education: Alternative Approaches of Online Learning during COVID-19 Pandemic Crisis
}

\author{
Dr Ankuran Dutta* \\ Department of Communication \& Journalism \\ Gauhati University, Guwahati, Assam, India \\ DOI: 10.29322/IJSRP.10.05.2020.p10169 \\ http://dx.doi.org/10.29322/IJSRP.10.05.2020.p10169
}

\begin{abstract}
Indian higher education can boast of being one of the torch bearers among many developing nations for its rich plethora of dynamic content. Due to the Novel Coronavirus (COVID-19) pandemic, in all the higher educational institutions, which includes universities, standalone institutes, and colleges, a total of about 10 million academic hours are compromised, which will be rather difficult to compensate. The University Grants Commission through its advisory instructed all the institutes to continue classes in online mode as per feasibility and engage ICT tools available for use in academic discourse. Many institutions have been using different social media platforms for the dissemination of knowledge. The present crisis has revolutionized the entire higher education architecture of the country through videoconferencing based online learning since there's no other option to compensate for the compromised academic activities. This paper aims at exploring the kind of social media used to disseminate learning resources to the students, and the impact it crafting on their educational loss. It also elucidates the effectiveness of online classes, e-learning pedagogy, and its outcome through structured qualitative analysis.
\end{abstract}

Index Terms- COVID-19, Novel Coronavirus, Pandemic, Indian Higher Education, Impact of Social Media, Online Learning, Videoconferencing, Digital Media, Teaching Pedagogy, UGC, ICT Initiative, Digital Repository, Open Educational Resources, Lockdown, Semester, Graduation and Post-Graduation Education.

\section{INTRODUCTION}

The institutional framework of higher education in India comprises universities, colleges and stand- alone institutes, where millions of students pursue diverse academic programmes. The higher education system in the country has grown exponentially in the length and breadth of the country, particularly in the post-independence period, and has stealthily grown into the largest system of its kind in the world. While this exponential growth is often hindered by shortcomings that include finance and management, including access, equity and relevance, reorientation of programmes by emphasising on health consciousness, values and ethics, and quality of higher education together with the assessment of institutions and their accreditation. These issues were evaluated by the University Grants Commission (UGC) through different deliberations since they lay a strong foundation for the education sector which is a powerful tool to build a knowledge-based information society of this century (Dutta \& Goswami, 2020).

The even semesters of almost all the higher education institutes start from January and the period between March and April is mid-semesters in different colleges and universities that are a crucial time for academic activities. Practically, in a semester, there are full-term classes only for a span of three to four months of a semester. But, due to sudden yet evasive coronavirus pandemic, all higher education institutions were compelled to shut down in between the mid-semester. This could possibly affect the higher education scenario in different ways. Having left with no options, a lockdown was the only possible way out to manage this tremendous crisis. Now, such a crucial step should be assisted with wise academic decisions, proactive enough to bail out an academic year of around 37 million students as well as give a roadmap for future prospects. To understand this, it would be pertinent for us to have a look at the present scenario of higher education in the country.

\section{Higher Education Architecture in India}

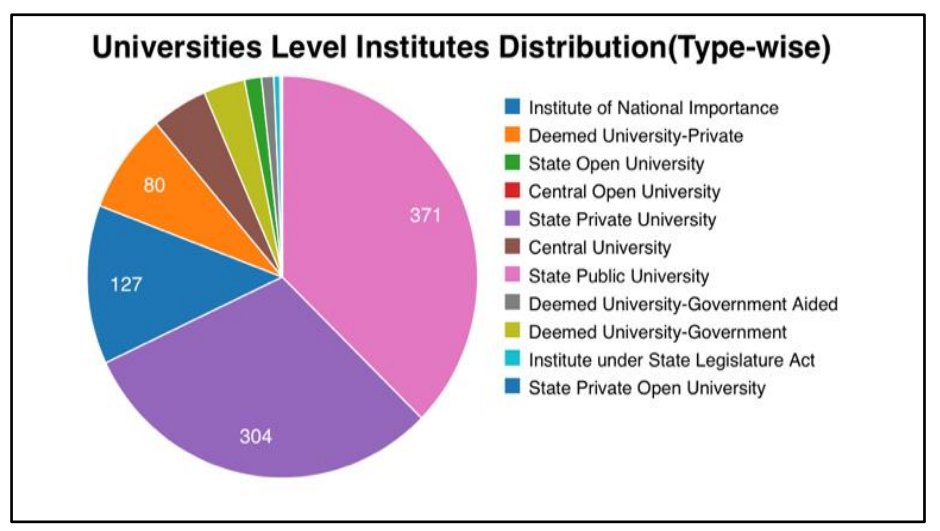




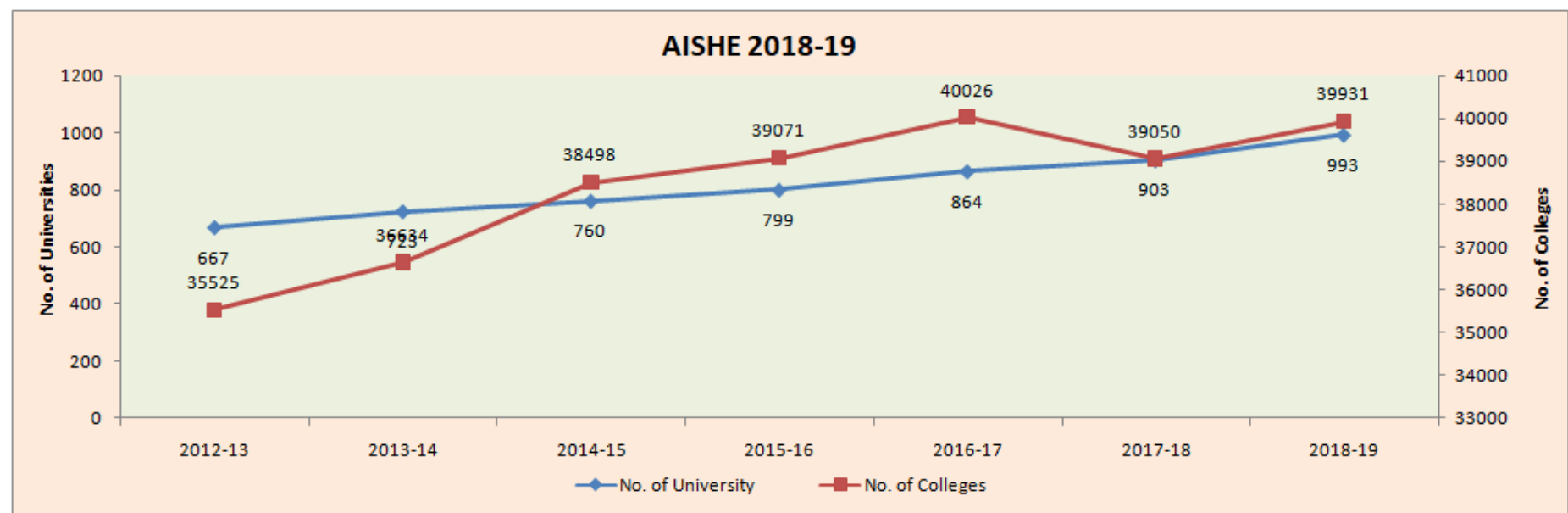

The All India Survey of Higher Education (2019) reveals the fact that Indian higher education comprises of 53,620 institutions with 37 million students. There is a total of 41,901 colleges, 10, 726 standalone institutions and 993 universities, out of which 385 Universities are privately managed and 394 Universities are located in rural areas. There are 548 General, 142 Technical, 63 Agriculture \& Allied, 58 Medical, 23 Law, 13 Sanskrit, and 9 Language Universities and rest 106 Universities belong to other categories. Out of 1.4 million teachers engaged in higher education in India, 1.1 million teachers teach in the colleges, 158,000 teachers in universities, and 147,000 teachers are employed in the standalone institutions. A total of 26 million students study in different colleges followed by 7 million students in universities and 2 million in the standalone institutions. Total enrolment in higher education has been estimated to be 37.4 million with 19.2 million male, and 18.2 million female, which constitutes $48.6 \%$ of the total enrolment. Gross Enrolment Ratio (GER) in higher education in India is $26.3 \%$, which is calculated for $18-23$ years of age group. GER for the male population is $26.3 \%$, and for females, it is $26.4 \%$. For Scheduled Castes, it is $23 \%$, and for Scheduled Tribes, it is $17.2 \%$ as compared to the national GER of $26.3 \%$ (AISHE, 2019).

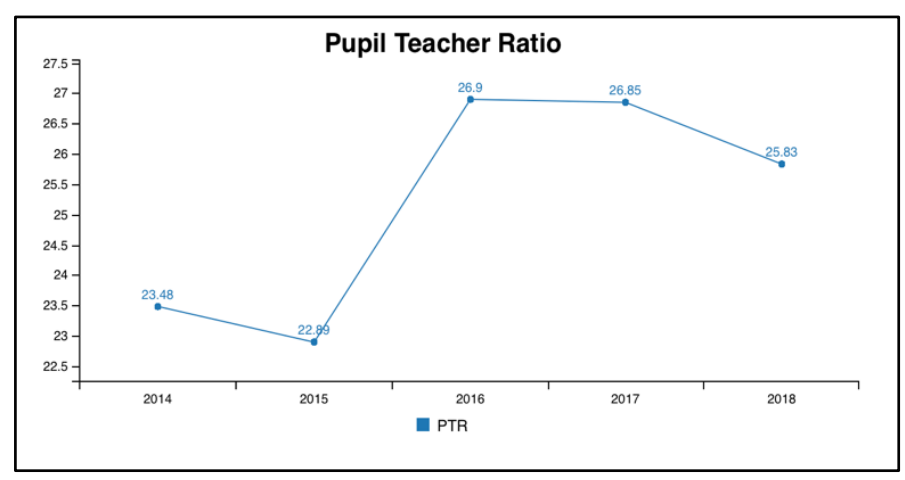

Furthermore, the report states in addition to one central open university, 14 state open universities and one state private open university, there are 110 dual mode universities, which offer education through both open and distance mode, and the maximum 13 of them are located only in Tamil Nadu. Distance enrolment constitutes about $10.62 \%$ of the total enrolment in higher education, of which $44.15 \%$ are female students (AISHE, 2019).

\section{Impact of Novel Coronavirus (COVID-19) in Indian Higher Education}

Due to the Novel Coronavirus lockdown of 40 days (first and second phase), in all the institutions, a total of about 10 million academic hours will be compromised, which will be rather difficult to compensate. The University Grants Commission through its advisory instructed all the institutes to continue classes in online mode as per feasibility and engage ICT tools available for use in academic discourse. The faculty members of the university and colleges are also requested by its advisory to make use of the various online tools in order to reach out to the students. To assist this endeavour, there are many open access tools available, which are already used by many universities and colleges to reach out to students located in remote areas. For example, many Departments of Gauhati University, Assam has been using different online tools, such as live online classes through Skype, use of YouTube with recorded academic and class lectures, readymade lectures through NPTEL, lectures through Google Classroom, live online classes through Piazza, Zoom, EasyClass, etc. With the availability of highspeed mobile networks, online live classes from home became a reality and many success stories have emerged in the process of engaging the students effectively using different online applications. Despite the fact that the attendance rate in the classes is not more than 80 percent in aggregate due to the poor network connectivity, it restricts a sizeable number of students to avail of these opportunities.

\section{Advisory to utilise online resources}

The UGC advisory has instructed the faculty members to develop online teaching content, such as instructional materials using modules, powerpoint presentations, recording video lessons, and even audio teaching materials that can be shared through different content sharing 
platforms and messaging applications like Whatsapp, Messenger, Imo, Viber, etc.

The UGC's recommendation for the faculty members and students has been to avail maximum benefit from various digital/ICT initiatives of MHRD, UGC, and its Inter-University Centres. Among the ICT facilities, SWAYAM is one of the most user-friendly MOOC (massive open online course) platforms. SWAYAM, stands for Study Webs of Active Learning for Young Aspiring Minds, which was initiated by Government of India and designed to achieve the three cardinal principles of education policy viz., access, equity and quality. The objective of this effort is to take the best teaching learning resources to all, including the most disadvantaged. SWAYAM seeks to bridge the digital divide for students who have hitherto remained untouched by the digital revolution and have not been able to join the mainstream of the knowledge economy. Swayamprabha is another ICT facility for viewing digital courses on television. The government facilitates different digital content through National Digital Library, e-PG Pathshala, Shodhganga, e-ShodhSindhu and Shodhgangotri. Accelerate hands on learning, the government initiated eYantra that creates a laboratory for training in embedded systems in collaboration with IIT Bombay. FOSSEE is a provision that allows access for use of open source software. Spoken Tutorial and Virtual Labs are also remarkable initiatives for self-training and virtual experiments.

The UGC has encouraged the faculty members to carry on research and write research articles during the nCoronavirus lockdown. Apart from the research based portals like Shodhganga, e-ShodhShindhu, the union government has an expert database and national research network called VIDWAN, Indian research information network system called IRINS and ShodhShudhhi, which is a plagiarism detection software to encourage the researchers to contribute original information by preventing plagiarism and maintain the reputation of Indian research. Although there are lots of open educational resource (OER) materials made available by the union government, a comprehensive and userfriendly online face to face teaching-learning ICT tool is an urgent requirement to reach out to the students who are located in the remote places (Dutta \& Goswami, 2020).

The recommendations set forward by the UGC have been visionary, provided the application of such facilities are widely accepted and applied by the teaching fraternity who have to understand that South Asian countries like China, South Korea, and Japan among others have not compromised on the teaching-learning time even at the wake of such crises. Also, there are risks of many students having to drop a valuable academic year due to extra months added to the course completion and others might simply engage in alternative areas.

\section{Research Elaborations}

\section{a) Statement of the Problem}

Due to the social distancing and Coronavirus lockdown in India, the higher education institutions were shut down in the mid of their academic semester, resulting in huge loss of knowledge diffusion and students' access to instructional activities that are designed in a face to face teaching-learning pedagogy. In view of this crisis, this study examines and assesses the impact of digital social media to reach and retain the academic activity of the students of higher education.

\section{b) Purpose of the Study}

This study was piloted to evaluate the impact of digital social media on higher education with special reference to the alternative teaching-learning approaches adopted by higher education institutions during nCoronavirus lockdown in the country. To be precise, the study aims at exploring the kind of social media used to disseminate learning resources to the students of higher education institutions in India, and the impact it crafting on their educational loss.

c) Objectives of the study:

- To identify what digital social media platforms have been used by the higher education institutes in India as an alternative approach to deliver learning components

- To identify the usage of digital social media by students in balancing the academic loss during COVID19 crisis

- To identify the impact of adopting social media practices in providing and disseminating normal academic knowledge

\section{d) Research Questions}

The paper intended to determine the impact of digital social media as alternative online learning strategies in higher education and determine the engagement of students in normal academic activities. To answer the question and analyze how this impact has taken place, the following research questions were considered:

RQ1. What are the popular digital social media platforms used by different higher education institutes in balancing the academic loss during the COVID-19 crisis?

RQ2. Do digital social media platforms have an impact on maintaining efficiency and interactivity among the students and the teachers?

RQ3. What are the benefits of using digital social media as an alternative online learning approach in academic knowledge dissemination? 
e) Significance of the study

An understanding of the use of digital social media, how it influences students' learning environment would be of great relevance as an alternative online learning approach during a crisis situation. The outcome of the study will undoubtedly draw a structure to strategize and reconstruct a new learning environment during crises and risks.

\section{f) Research design}

In the empirical part of this study, a qualitative approach was employed using a semi-structured interview among some selected students of three universities in India. Qualitative research methodology was used as it is appropriate to collect data in a natural setting rather than a contrived situation (Creswell, 2007), in this study, telephonic interviews between the researchers and participants were employed to avoid human bias whenever possible. Therefore, the researchers in this study were able to discover detailed information on how students are using and benefiting from social media through in-depth interviews. A total of 15 interviews were conducted along with three focus group discussions from the selected three universities. For the FGD, the Zoom and Skype platforms were used and a total of 18 students participated in three sets of meetings. In addition to the in-depth telephonic interview and three online FGDs, 67 feedbacks were also collected from the students participating in the online classes. The feedbacks were based on unstructured questions with openended responses which would produce qualitative data. These open-ended questions allowed the respondents to express and responded to the questions in-depth with their own words instead of the words fed by the researcher.

The collected data was transcribed and categorized based on the answers of the participants. Relevant themes and coding were developed from the transcriptions. The data of this study were analyzed inductively, starting with the raw data consisting of multiple sources of information and then broadening to several specific themes. Also, key words were written down on the margin and then grouped the margin notes into different subthemes and themes.

\section{Findings and Analysis}

Based on the analysis of qualitative coding, the findings were categorized under five major heads- impact of the lockdown, provision of online classes, pedagogy adopted in the teaching learning process, benefit and limitations of online classes.

a) Impact of Lockdown: A lockdown is an emergency protocol that prevents people from leaving a given area. A full lockdown will mean everyone must stay where they are and not exit or enter a building or the given area. This scenario usually allows for essential supplies, grocery stores, pharmacies, and banks to continue to serve the people. All non-essential activities remain shut for the entire period (ET Online, 2020). In India, the lockdown to curve the scale of novel coronavirus was initially given for 21 days, which was later extended to another 19 days until 3 May, 2020. The isolation and fear in this lockdown era are driving an escalating nervousness among many people. Not just those with pre-existing conditions like anxiety, depression, and OCD (obsessive-compulsive disorder), but also the elderly who are struggling with new levels of loneliness and helplessness. The psychological impact of lockdown or quarantine exposed proof of a range of psychological conditions, from post-traumatic stress symptoms to confusion, anger, depression, stress, insomnia and emotional exhaustion (Rahman, 2020).

During the data collection, it was observed that about $80 \%$ of the students, who were interviewed and feedback was taken have been suffering from excessive stress, depression and anxiety. A student commented, "Lockdown boredom and anxiety covered me up". Facing extreme uncertainty, a master's student stated, "It often lead to frustration and I would end up having arguments at home. Brainstorming wasn't doing good. That is why I stopped thinking about any of it". Another master's student added, "Being an extrovert, in this quarantine time, it's very difficult being stuck in a square size room and spending the whole day without doing anything productive". Within an overnight notice, even before the lockdown started in India, the students had to leave their hostels and goback to their homes apprehensively They became very helpless in that situation, they couldn't even discern how long the cancellation of the regular classes would continue. Many students even didn't bring their books, essential gadgets like laptops as they considered the lockdown period as a short-term vacation. However, the lockdown is yet to be lifted and may continue for a longer duration of time. Hence, it remains uncertain, whether; the students will be able to go to their universities during this academic session. In particular, the final semester students, who are supposed to submit their projects and dissertations have been feeling very stranded, since, they are unable to collect primary data from the field.

b) Provision of Online Classes: Online learning is defined as learning that takes place partially or entirely over the Internet. This definition excludes purely print-based correspondence education, broadcast television or radio, video conferencing, videocassettes, and stand-alone educational software programs that do not have a significant Internet-based instructional component. There are two purposes for online learning: learning conducted totally online as a substitute or alternative to 
face-to-face learning and online learning components that are combined or blended (sometimes called "hybrid") with face-to-face instruction to provide learning enhancement (Means et al., 2010). Due to the uncertain novel coronavirus menace, the higher education system in India became handicapped overnight. With the initiative at the institutional level and teachers' personal efforts, almost all the universities and higher learning institutes in the country started online classes in place of face to face traditional classes. Apart from the open universities and the distance education directorates of the dual-mode universities, many conventional institutes offered online classes to make the education continue and complete the curricular in this semester. A student credited the effort of online classes as, "Our online classes were not only about studies but also about our mental health'. Another master's student stated, "Even when the world is scientifically paralysed at the moment to dig out a solution to counter the pandemic coronavirus disease, online classes have been an innovative and digitally equipped medium to save and help every student to add their efficiency and upgrade their educational skills". These online classes in place of the traditional face to face classes became very useful not only for the educational advancement but, also provided great assistance to their mental health. Hence, in a feedback, a student mentioned, "It helped not to broke in this mental situation." A post graduate student also added, "We were given tips on how to keep ourselves stress free and occupied with fruitful things".

c) Teaching Pedagogy: Pedagogy is defined simply as the method, and practice, of teaching. It is the art and act of teaching to make learners to get knowledge and to acquire skill based experience. Watkin and Mortimer (1999) define pedagogy as any conscious activity by one person designed to enhance the learning of another. Akhilesh Arvind Nimje and Kalyani Tripathi Dubey (2013) examined the Socratic lecture model on the teaching pedagogy in changing educational scenario and concluded that the Socratic lecture model allows the teachers to teach in a very smooth way. In general, pedagogy refers to the method in which a teacher teaches to make their classes more attractive and useful. It is the theory and practice of educating. Pedagogy is the relationship between learning techniques and culture and is determined based on an educator's beliefs about how learning should, and does, take place. Pedagogy requires meaningful interactions by establishing mutual respect between educators and learners. The goal is to help students build on prior learning and develop skills and attitudes and for educators to devise and present curriculum in a way that is relevant to students, aligning with their needs and cultures (Persaud, 2019). During this pandemic crisis, the educators and more particularly the teachers of the higher education institutes were bound to design their pedagogy to engage the students. Some of the pedagogies were not based on any theory, but they were innovative. The teachers, who were literally challenged in using ICT for education also became experts in providing online teaching or using different social media platforms to engage their students academically and mentally. While discussing informally with a few university professors, the researcher found that providing meaningful teachings, creating an academic environment during quarantine by adopting new technologies is their accountability to the profession and responsibility towards their students. Even they denied receiving any instructions from their authority to conduct the classes except an advisory, they initiated the online classes only to help their students. The following is an alternative arrangement of face to face classroom teaching adopted by an academic department of an Indian public university.

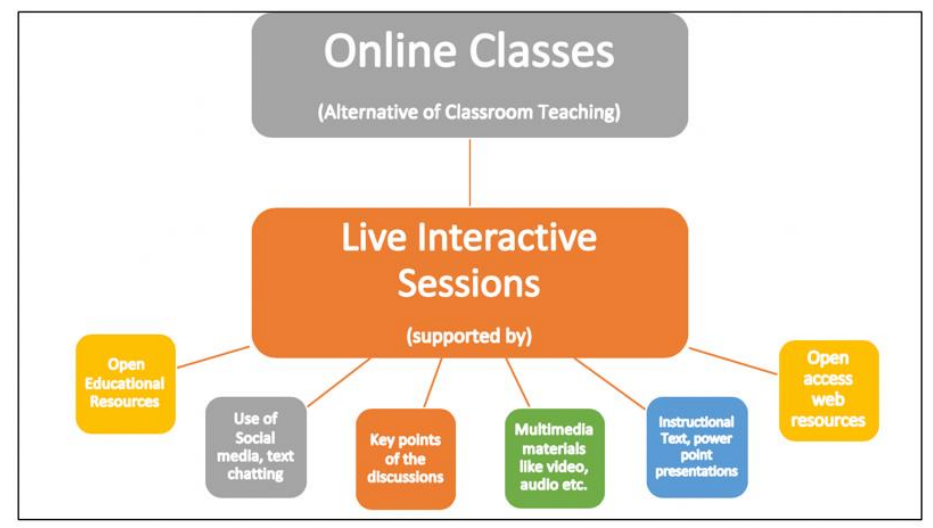

a) Benefits of Online Classes: Online learning has become an indispensable part of academic and professional education during this novel coronavirus pandemic. The University teachers have been exploring to create a more appealing and effective online learning environment. Lots of benefits have been mentioned by the participants during the qualitative data collection. A master's student expresses gratitude to the teachers saying, "The government officials are saving us today by doing every possible thing and our teachers are preparing us for tomorrow for what comes next". Another student has articulated the benefits from the online classes as, "The online classes have been very fruitful during these difficult times. Firstly, during this lockdown the online classes provide us a daily purpose to attend it, which helps us in staying somewhat focused and keeps us in the correct lane. Secondly, the syllabus is getting covered during these off-days, as there will be hardly few days (hopefully) for the completion of the syllabus in the future. Moreover, it is a unique experience which we are having and it certainly brightens us with hope for the 
upcoming days". In a FGD, a participant said that apart from the curriculum based learning, she learnt how to handle a crisis from the online classes. Another participant stated the online classes helped her to maintain the flow of study. Among the participants, a student said, "The assignments and study materials given by the teachers are enhancing my understanding of the subjects and is also helping me to make myself busy in this duration".

Providing the feedback, a post graduate student of Mass Communication highlighted the benefits of online classes as, "The online classes have been really helpful to me during this lockdown period, for which I can keep myself engaged with all my studies or academic syllabus; it keeps me away from experiencing negativity, boredom or uselessness. Yes, there are occurrences of some technical issues at times, but, apart from that it is fine. The teachers are trying to cover the syllabus during this lockdown and are keeping us engaged with our studies by giving assignments and study materials like pdf files, PPTs etc. I am really enjoying the online classes and I really thank all the teachers, who are being extremely supportive all the time". It's evident from the focus group discussion, feedback and in-depth interviews that there are lots of advantages of using online classes, especially along with the academic activities; engagement through this mode helped the students' mental health.

b) Limitation of Online Classes: For any approaches, if there are some benefits or advantages, a few limitations will be there too. The students are accustomed to the face to face classroom learning and hence, some of them couldn't adjust themselves with the new academic environment while staying at home. There are pros of classroom teaching that digital or online education will find hard to replace, such as collaborative learning, enhancement of critical thinking skills, improvement of social skills, building organisational skills, keeping students stimulated, development of important personality and career building skills (Singhal, 2017). According to a participant, "I'm really struggling with my online classes, I know if I want to graduate I have to complete them, but, it's obvious I do better in a classroom environment. Suspending our classes was the right thing to do but it's tough". Lots of advantages are missing in the online classes. However, in this research, it was found that these issues are not vital for the students. The basic limitation is the poor internet connectivity and mobile data. The final semester students of the universities are more emotional since they have only a couple of months to complete their master's programme and hence, they are terribly missing the last days of a classroom environment. Also, another issue of mobile data was highlighted, "After the online classes are over, there is no Internet data remaining for completing other assignments or for even Google search".

A second semester post graduate student opines, "Online classes haven't been in any way helpful to me because I couldn't attend a single classes properly due to network issues. With the initial assignments, I took help from others' assignments to understand what I was asked to do, but, after a few assignments I was unable to do the same. Without understanding I am not being unable to do anything. It is somehow affecting me very adversely badly. I feel I am lagging behind while the rest of the people students have been progressed. I kept thinking of how I am not being unable to do anything about it since there is no one to blame and I also didn't don't want to look like an excuse giver. It often leads to frustration and I would end up having arguments at home. Brainstorming wasn't isn't doing any good either. That's is why I have stopped thinking about any of it. The classes are not helping me at all". While discussing with a couple of teachers, it was their opinion that if the $80 \%$ students are taking the benefits, they shouldn't stop the initiatives for the rest of $20 \%$. Once, everything becomes normal, teachers need to give extra tutorials for the rest of $20 \%$ students. A few students have also mentioned in their feedback that although they have mobile internet connectivity, they always get in touch with the rest of the students and collect the recorded discussion of the online classes and submit their assignments on time. In spite of poor internet connectivity, a student expressed his spirit, "At times we have to face the potholes of network but that doesn't let down our spirits".

\section{Discussion}

The above findings are from the focus group discussion, feedbacks, and telephonic in-depth interviews collected from a set of selected students of three Indian public universities. It's visible that the teachers and students both have been continuously engaged in searching good alternatives for the dissemination as well as the reception of the academic knowledge during the pandemic menace. Let's enumerate the research questions from the findings and results of this qualitative analysis.

$R Q 1$. What are the popular digital social media platforms used by different higher education institutes in balancing the academic loss during the nCoronavirus crisis?

It's found that different social media applications have been used by the students for acquisition of academic knowledge. However, the popular social media sites, like Facebook, Twitter, and Instagram are used only to collect 
information, not necessarily pertaining to academic knowledge. Meanwhile, social media allows students to develop communities to share experiences, discuss conceptions, and create a space for co-learning. Educators can interact with students beyond the confines of the classroom, too (Persaud, 2019). Among different messaging applications, in India, WhatsApp has been used mostly to share information, documents, presentations, texts, videos, etc. YouTube has become one of the most significant tools for self-learning. Video conferencing applications such as Zoom, Skype, Jitsi Meet Cisco Webex, Starleaf, Whereby, Hangouts, etc have become highly popular tools for keeping in touch with students in a world which is increasingly getting socially isolated during the coronavirus pandemic. A student has mentioned his experience of using Skype for the online classes, "You get the intensity of something when you go through a phase, experience and deal with it. I had heard about the 1918 Spanish Flu that infected 500 million people and killed an estimated 10-50 million, devastating the global economy. Something similar would hit us was never in my thoughts. I still cannot swallow the fact that on 14th March of the varsity phase was the last day we were out free, enjoying, and now here we stand without any certainty as to when we see our department again. I, honestly, didn't imagine that online-classes through Skype will fill up that distance and make our days pass with ease. I am fortunate that our teachers had taken this initiative to keeps us occupied to live a mentally-sound time during selfquarantine".

\section{RQ2. Do digital social media platforms have an impact on maintaining efficiency and interactivity among} the students and the teachers?

To get in touch with the teachers and the classmates, the students who were not previously tech-savvy, they are also using different social media tools now. A student in her feedback mentioned, "In these turbulent times when the whole world is wishing for a miracle us students have already found one. Thanks to our teachers, online classes have been a great outlet for us. Having being able to connect with our teachers and friends through technology has been a boon for us. Technology has been created to make life better and easier, I believe we are fully utilising its potential. Lockdown and self-isolation have taken a toll on us all but online classes have been really fun and educative." Apart from the text messengers, video conferencing tools have been widely used for interactions between the teachers and students. Students are now comfortable using technology to help them learn, and to access, share, and create useful information and gain knowledge in a subject. Digital social media has broken the monotony of the classroom teaching as the students can now access relevant multimedia materials like videos, lecture notes, presentations, and overall wider access to sources and experts online.

\section{RQ3. What are the benefits of using digital social media as an alternative online learning approach in academic knowledge dissemination?}

In online classes, the poor network connectivity in the fringe areas creates a huge problem, even in the middle of the classes; the connection gets disconnected due to network problems, system error, software updates, etc. Although, there are technical glitches in using social media as an alternative online learning approach, it's useful for nearly $80 \%$ of the student participants in the study. A student says, "If I say about the online class honestly this is the first experience for me and I am really glad to have this opportunity. It's been six years for me of staying away from home and whenever I visit home, I always bring some books with me but could not concentrate even for a while. But this time these online classes have been able to connect me with my academic work". Another student remarked, "I believe online classes have paved a way for technology to be a friend in taxing times and aids to prevent the disruption of regular classes which otherwise would have had dire consequences on a student's life. It bridges the gap between the inaccessibility of transportation and education, thereby, providing an outlet to learn, interact, and disseminate information". The technical glitches hamper the spirit of online learning in a country like India. Nevertheless, the authorities of the higher learning institutions are bound to bridge the gap; educators and teachers are accountable to their students during this crisis and students are compelled to retain education for completion of their academic programmes.

\section{Conclusions}

In a world where digital social media has taken a significant role in teaching and learning, any modern pedagogy much accounts for students' findings, analysing, and applying knowledge from a growing number of constantly changing sources. This requires higher-order skills like critical thinking and the ability to learn more independently, as well as in larger groups, both in person and online (Persaud, 2019). This study focused on the impact of digital social media on student academic life in higher education during the Novel Coronavirus (COVID19) pandemic crisis.

The study explains the impact of self-isolation, quarantine and lockdown due to the pandemic on the academic schedule of the students of Indian higher education. After studying the phenomena that are of interest to the study, and transcribing the various responses of the participants, even though some of the responses were not transcribed because 
they were all communicating the same idea; the results reveal that social media is widely used by students of higher institution. Opportunities and benefits of digital social media for academic purposes incurred by the students became visible only after the crisis took place. Although digital education based online learning was not systematically implemented in India, even in the open and distance learning as a whole, because it was not an essential pedagogy until the pandemic struck the world and the Indian state in particular. The present Coronavirus crisis has revolutionised the entire higher education architecture of the country through e-tools for teaching and learning, since there's no other option for continuation of the academic activities. There are some limitations of this study, considering the number of participants involved and the methodology adopted due to the COVID-19 lockdown in India.

Nevertheless, the success of the use of digital social media as an alternative of the face to face classroom teaching depends on the commitment of the faculty members of higher educational institutes and how they consider their role in such a situation when individuals engaged in other occupations like doctors, other health workers, police, administrators, journalists are waging a war against the virus, the teaching fraternity is required to equip the new age students with knowledge and teach them the value of time. It is only responsibility and accountability to the service that can enable teachers to engage their students by providing quality instructions to utilise this lockdown period because nobody knows for how long the lockdown extends in the country and how socially and economically it could impact the lives of many. This article concludes quoting feedback of a student, "The phrase "hope for the best and prepare for the worst' is what we are learning every day".

\section{References}

[1] Dutta, A., \& Goswami, A. L. (2020, May 4). Corona Pandemic and Higher Education. The Assam Tribune, p. 4.

[2] ET Online. (2020, March 25). What a coronavirus lockdown looks likes, and what you can do \& what you can't. The Economic
Times. https://economictimes.indiatimes.com/news/politic s-and-nation/coronavirus-outbreak-what-a-lockdown-willlook-like-for-you/articleshow/74760719.cms?from=mdr

[3] Means, B., Toyama, Y., Murphy, R., Bakia, M., \& Jones, K. (2010, September). Evaluation of Evidence-Based Practices in Online Learning: A Meta-Analysis and Review of Online Learning Studies. U.S. Department of

Education. https://www2.ed.gov/rschstat/eval/tech/evidence -based-practices/finalreport.pdf

MHRD. (2019). All India Survey of Higher Education 2018-2019. Department of Higher Education, Ministry of Human Resource Development, Govt. of

India. https://aishe.gov.in/aishe/viewDocument.action?docu mentId $=262$

Nimje, A., Dubey, T. (2013), The Socratic Lecture Model: An Effective Teaching Pedagogy in Changing Educational Scenario, IOSR Journal of Humanities And Social Science (IOSR-JHSS), Volume 14 , Issue 6 (Sep. - Oct. 2013), pp. 117-121

Persaud, C. (2019, March 1). Pedagogy: What educators need to know. Top Hat. https://tophat.com/blog/pedagogy/

Rahman, A. P. (2020, April 4). Isolation and mental health: The psychological impact of lockdown. The Hindu. https://www.thehindu.com/society/isolation-andmental-health-the-psychological-impact-oflockdown/article31237956.ece

Singhal, S. (2017, July 5). 7 ways classroom teaching is better than online education. India Today. https://www.indiatoday.in/educationtoday/featurephilia/story/classroom-teaching-better-thanonline-education-984387-2017-06-23

Watkins C and Mortimer P (1999). Pedagogy: What do we know? In Mortimer $\mathrm{P}(\mathrm{Ed})$, Understanding pedagogy and its impact on teaching. (pp 1-19) London: Chapman.

\section{AUTHOR}

The author is an Associate Professor and Head, Department of Communication and Journalism, Gauhati University, Guwahati, Assam, India. Dr Dutta is a media educator by profession with an experience of about 15 years and involved in different academic activities in India, Bangladesh, Canada, Maldives, Sri Lanka, Pakistan and Malaysia. $\mathrm{He}$ is a recipient of "National Award for Outstanding Efforts in Science and Technology Communication, 2018" of NCSTC, Govt. of India. He also serves Dr Anamika Ray Memorial Trust as Managing Trustee. Email: ankurandutta@gmail.com 